\title{
Peplography: an image restoration technique through scattering media (Withdrawal Notice)
}

Myungjin Cho, Ki-Ok Cho, Youngjun Kim

Myungjin Cho, Ki-Ok Cho, Youngjun Kim, "Peplography: an image restoration technique through scattering media (Withdrawal Notice)," Proc. SPIE 9867, Three-Dimensional Imaging, Visualization, and Display 2016, 986717 (1 June 2016); doi: $10.1117 / 12.2228567$

Event: SPIE Commercial + Scientific Sensing and Imaging, 2016, Baltimore, MD, United States 


\section{Peplography: an image restoration technique through scattering media (Withdrawal Notice)}

Proc. SPIE 9867, 986717 (2016)

Online Publication Date: 1 J une 2016

Withdrawn from Publication: 12 August 2016

Conference Date: 18-20 April 2016

Conference Location: Baltimore, Maryland, United States

Conference Title: Three-Dimensional Imaging, Visualization, and Display 2016

Conference Chairs: Bahram Javidi, Jung-Young Son

Myungjin Cho, Ki-Ok Cho, Youngjun Kim

Hankyong National Univ. (Korea, Republic of)

Publisher's Note: This paper, originally published on J une 1, 2016, was withdrawn at the author's request on August 12, 2016. (c) (2016) COPYRIGHT Society of Photo-Optical Instrumentation Engineers (SPIE). 